\title{
THE NATURE OF THE CHEMICAL ANOMALIES OF THE SIRIUS A
}

\author{
V. LEUSHIN \\ Special Astrophysical Observatory of RAS, Russia \\ leushin@sci.lpi.msk.su
}

On the basis of the analysis of the observed equivalent line widths of FeI, FeII, and TiII in the spectra of Sirius A and Omicron Peg and calculation of abundances of these elements, the oscillator strengths of the lines used are refined. With the improved oscillator strengths, the iron and titanium abundances in the atmosphere of Sirius A are obtained with a higher accuracy than previously:

$$
\begin{aligned}
& \operatorname{lgN}(\mathrm{FeI})=7.899 \pm 0.011, \\
& \lg \mathrm{N}(\mathrm{FeII})=7.908 \pm 0.010, \\
& \operatorname{lgN}(\mathrm{TiII})=5.30 \pm 0.02 .
\end{aligned}
$$

The improved accuracy allowed one to conclude that the surface magnetic field is absent in the atmosphere of Sirius $\mathrm{A}: \mathrm{H}=3 \mathrm{D} 0 \pm 100 \mathrm{Gs}$. The equivalent widths of 7 helium lines of the red region of the spectrum are measured. The calculations of these lines (with allowance for their blending with lines of other elements) show normal helium abundance. Following a comparison of features of Sirius A and Omicron Peg, a suggestion is advanced on possible reasons for the existence of Am stars. 